\title{
Some novel aspects of DNA physical and chemical gels
}

\author{
Diana Costa $^{\text {a,b** }}$, Salomé dos Santos ${ }^{b}$, Filipe E. Antunes ${ }^{\text {a,b }}$, Maria G. Miguel, \\ and Björn Lindman ${ }^{\text {a,b }}$ \\ ${ }^{a}$ Physical Chemistry 1, Centre for Chemistry and Chemical Engineering, Lund University, Box \\ 124, S-22100 Lund, Sweden \\ ${ }^{b}$ Department of Chemistry, University of Coimbra, Coimbra, Portugal \\ E-mail: diana.costa@fkem1.lu.se
}

Contribution in honour of Professor Armand Lattes

\begin{abstract}
DNA chemical and physical networks have been investigated with respect to their stability and swelling, and rheological properties. Chemical networks were prepared by cross-linking DNA with ethylene glycol diglycidyl ether (EGDE), and physical networks by association with cationic polymers. Both types of gels display strong elastic properties and have a shear thinning behaviour. Addition of cationic surfactants effectively collapses the chemical gels, de-swelling starting from a critical aggregation concentration (cac) much lower than the critical micelle concentration $(\mathrm{cmc})$ but similar to that for binding of surfactant to DNA in solution. The swelling-deswelling process appears to be reversible; thus the addition of an anionic surfactant to a gel collapsed by cationic surfactant gives a gel volume close to that of the original gel.

Physical networks prepared by mixing DNA (either single- or double-stranded) with cationic polyelectrolytes, both derivatives of hydroxyethyl cellulose, one of them carrying hydrophobic groups, show an intriguing asymmetric phase separation and a very different rheological response from that of the polymers alone. Phase maps of the mixtures show three distinctive regions, a two-phase region, a bluish one-phase region and a transparent one-phase region. Effects due to hydrophobic groups on the polymers are relatively minor.
\end{abstract}

Keywords: DNA, cross-linking, chemical gels, physical gels, swelling-deswelling

\section{Introduction}

Polymer gels are central in both colloid science and polymer science, and are common in biological systems and used in many technological applications. The three-dimensional networks are stabilized by crosslinks, which may be provided by covalent bonds or by physical 
interactions, such as charge transfer complexes, hydrogen bonding, van der Waals interactions, electrostatic or hydrophobic interactions. ${ }^{1}$

Polymer gels that respond to changes in the surrounding environment with a volume transition, often referred as responsive gels, have attracted much interest in the last few years. ${ }^{2-9}$ In this group are the polyelectrolyte gels, which consist of charged polymer networks, counterions and solvent and are usually synthesized by chemically cross-linking charged or titrating polymers. The environmental conditions include $\mathrm{pH},{ }^{7,10-12}$ solvent composition, ${ }^{13}$ ionic strength, ${ }^{14}$ temperature, ${ }^{15,16}$ pressure, ${ }^{17}$ buffer composition, chemicals, ${ }^{18}$ photoelectric stimuli ${ }^{19}$ and photoirradiation. Because of their significant swelling and syneresis in response to external stimulation, these polymeric networks have a variety of applications such as contact lenses, ${ }^{20}$ diapers, wound dressing, membrane materials, pharmaceutical products, ${ }^{21}$ monolithic drug delivery systems, ${ }^{19}$ chromatography packing materials and agriculture. ${ }^{22}$ Moreover, cross-linked gels have been investigated for many biomedical uses as tissue culture substrates, ${ }^{23}$ enzyme activity controlling systems, ${ }^{24}$ materials for improved biocompatibility ${ }^{25}$, in the design and analysis of artificial muscles and biosensors and in the design of intelligent controlled drug release devices for site-specific drug delivery.

Of biological polyelectrolytes, DNA has attracted particular interest and there are numerous studies of the interactions between DNA and polycations. Positively charged agents interact predominantly by electrostatic interactions with DNA molecules and induce DNA compaction, aggregation and precipitation. Other studies of DNA condensation have been performed with liposomes ${ }^{26,27}$ and other oppositely charged components, like chitosans. ${ }^{28}$ Theoretical work concerning simulations of DNA/polycation systems are performed in order to understand confinement and/or the interactions between the oppositely charged molecules. ${ }^{29,30}$

Here we report some initial studies involving chemical and physical DNA gels. The preparation of physical gels from mixing DNA and cationic polyelectrolytes has been examined. The study is focused on such mixtures, which can find use in the thickening of DNA solutions. We were also interested in the difference in phase behaviour of the cationic polyelectrolyte with double stranded and single stranded DNA, which might bring the possibility of easily separating dsDNA from ssDNA. On the other hand, the chemically cross-linked DNA may find uses in medicine as drug delivery systems, and drug control during administration. Also, future development in the use of cross-linked DNA gels in local gene delivery applications seems to be a great challenge. Furthermore, these gels could be useful for separation purposes and also as a tool for investigating DNA-cosolute interactions by simply monitoring volume changes.

\section{Experimental Section}

Preparation of Chemical DNA Gels. Double stranded DNA, from salmon testes, was dissolved in water containing $3.7 \mathrm{mM} \mathrm{NaBr}$, with a DNA concentration of $9 \mathrm{wt} \%$. After mixing over night at $35^{\circ} \mathrm{C}$, the cross-linker (ethylene glycol diglycidyl ether, EGDE) was added and the solution 
was submitted to the vortex for 45 minutes. After adding $1 \mathrm{M} \mathrm{NaOH}$ and TEMED $\left(\mathrm{N}, \mathrm{N}, \mathrm{N}^{\prime}, \mathrm{N}^{\prime}\right.$ Tetramethylethylenediamine), the sample was mixed for another 10 minutes, and then transferred to test tubes and incubated for 2 hours in a water bath at $30{ }^{\circ} \mathrm{C}$. Gels with $1 \%$ and 3 $\%$ cross-linker were prepared in the same way. Freshly synthesised gels were neutralised and rinsed in $1 \mathrm{mM} \mathrm{NaOH}$ solution. The DNA gels swelled considerably in the $\mathrm{NaOH}$ solution and due to this fact the DNA concentration in gels is lowered. The concentration of DNA in gels equilibrated with $1 \mathrm{mM} \mathrm{NaOH}$ (reference state) was obtained by weighing gels before and after freeze-drying. A decrease in the DNA concentration from $9 \mathrm{wt} \%$, at preparation time, to around $1 \mathrm{wt} \%$, after immersion of the gels in the $\mathrm{NaOH}$ solution, was observed. Surfactant/gel samples were prepared by placing cylindrical gel pieces of known mass (1-3 g) into solutions of a cationic surfactant like cetyl trimethylammonium bromide (CTAB) and anionic surfactant, sodium dodecyl sulfate (SDS), at different concentrations. All samples were equilibrated in sealed containers for four weeks at $25^{\circ} \mathrm{C}$ and shaken slowly several times during that period of time.

Preparation of Physical DNA Gels. Cationic hydroxyethyl celluloses based polymers were used; one of these (JR400, denoted here $\mathrm{P}^{+}$) has a relatively high charge density and no hydrophobic groups, and the other (LM200, denoted $\mathrm{HMP}^{+}$) has a lower charge density and hydrophobic groups (dodecyl chains). The two polymers were mixed with both single and double stranded DNA. Polyelectrolytes were thus physically cross-linked by association with oppositely charged polymers. The gels formed were investigated with respect to rheological properties as well as thermodynamic stability. The latter was achieved by phase map determination; several samples were individually prepared by mixing DNA stock solutions with the desired amount of cationic polyelectrolyte and adding water (making a dilution of DNA stock solution to the desired concentration). After approximately 5 minutes in the vortex mixer, the samples were placed in a platform stirrer and after 24 hours they were centrifuged at $4000 \mathrm{~g}$ for about 2 hours. They were then placed again in the platform stirrer for another 24 hours, to ensure complete homogeneity. The phase map was determined by visual inspection. The samples were analysed several weeks after preparation and samples giving no phase separation were considered monophasic. All solutions were prepared with Millipore water performed at $25^{\circ} \mathrm{C}$.

\section{Results and Discussion}

A reproducible protocol was established for preparing DNA gels by cross-linking DNA, from salmon testes, with ethylene glycol diglycidyl ether (EGDE), which is a bifunctional cross-linker (the cross-linker has an epoxide structure) binding to the guanine bases of the DNA molecule. Gels with different cross-linking densities $(1 \%$ and $3 \%)$ were prepared following the normal procedure of gel formation. Experiments were mainly carried out with cylindrical gel pieces of known mass (1-3 g) with the crosslinking density of $1 \%$. 
All cross-linked DNA gels in their swollen state, in equilibrium with $1 \mathrm{mM} \mathrm{NaOH}$, were clear and transparent and had the same refractive index as water.

\section{Chemical DNA gels-Rheology}

Covalent DNA gels are strongly shear-thinning (or pseudo-plastic) systems as illustrated in Figure 1. The viscosity decreases with increasing shear rate and, as can be seen, there is a small plateau at low shear where the viscosity is independent of shear rate. In addition, Figure 2 presents the mechanical spectra with the storage modulus $G^{\prime}$ and loss modulus $G^{\prime \prime}$ plotted logarithmically against the frequency.

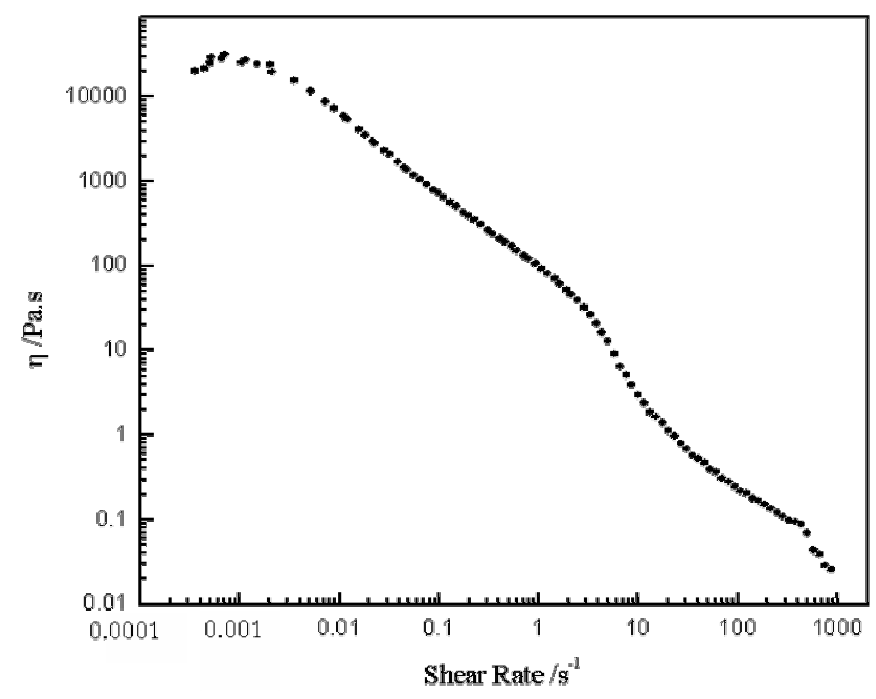

Figure 1. Dependence of viscosity on the shear rate demonstrates a shear-thinning behavior for chemical DNA gels.

The elastic modulus of DNA gels is 1-2 orders of magnitude larger than the viscous modulus and is almost frequency-independent, with the complex viscosity decreasing with frequency. DNA gels correspond to a good approximation to an elastic solid, in the frequency range studied. Such strong elastic properties found for other polymers have many applications in various fields of biomedical research, particularly in dynamic pressure stimulation in tissue engineering. Agarose gels, for example, due to their good elastic properties and availability, have been selected as the phantom material in magnetic resonance elastography (MRE) phantom study. ${ }^{31,32}$ 


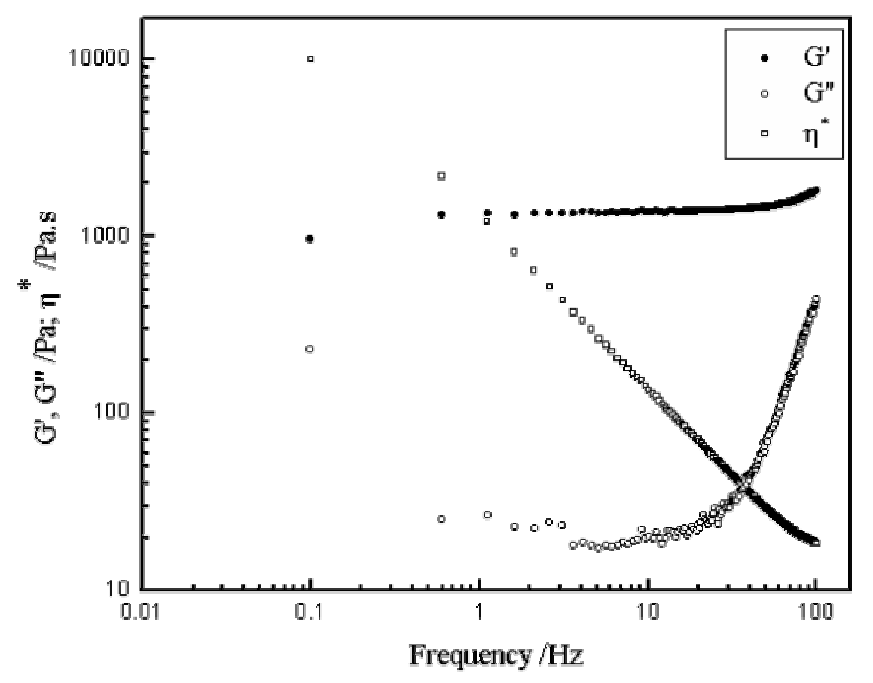

Figure 2. Storage, loss moduli and complex viscosity $\left(\mathrm{G}^{\prime}, \mathrm{G}^{\prime \prime}\right.$ and $\eta^{*}$ respectively) as a function of frequency for a chemical DNA gel sample. Rheological properties are presented as a function of frequency.

\section{Chemical DNA gels - swelling-deswelling behaviour}

Besides the intrinsic interest in covalent polymer gels, they can also be useful for monitoring interactions between the polyelectrolyte and different cosolutes. As shown in Reference 8, the changes in gel volume directly reflect the interactions between a polyelectrolyte and an oppositely charged cosolute. Like for other covalent polyelectrolyte gels, the chemical DNA gels show strong osmotic swelling; addition of electrolyte leads to a progressive gel shrinking. Addition of cationic surfactants gives, as exemplified in Figure 3, a dramatic deswelling starting at the concentration for onset of surfactant binding (critical association concentration, cac). The concentration for onset of deswelling is closely the same as that of onset of binding of surfactant to DNA in homogeneous solution and can be interpreted in terms of polyelectrolyte-induced surfactant micellisation. These findings will be detailed in a separate publication. ${ }^{33}$ We report here also on the effect of the addition of anionic surfactant to collapsed DNA gels illustrating the reversibility of the swelling process. This reversibility of the swelling of chemical DNA gels was studied by the addition of different concentrations of a cationic surfactant, CTAB, followed by the addition of different concentrations of an anionic surfactant, SDS. Figure 3 shows the interesting behaviour when collapsed DNA gels were immersed in SDS solutions. The most important aspect is that the relative volume $\left(\mathrm{V} / \mathrm{V}_{0}\right)$ returned to between 90 and $100 \%$ of the initial state. Thus the swelling of DNA gels appears to be reversible with a relatively minor hysteresis since the data points $\mathrm{V} / \mathrm{V}_{0}$ for the addition of SDS are slightly higher than the data points for the addition of CTAB. We argue that, as in our previous studies of the behaviour of DNA in the presence of a mixed cationic-anionic surfactant system ${ }^{34}$, the interaction between the two surfactants is stronger than that between cationic surfactant and DNA. In this case the 
response of the gels comes from the coil-globule transition ${ }^{34}$ of the polymer in solution, which is manifested as the swelling-shrinking transition in the gel form. This dynamic deswelling/swelling process could be very convenient in biological applications due to the possibility of the modulation of drug release.

\section{Physical DNA gels-Phase behaviour}

In this our initial study of physical gels in mixed solutions of DNA and cationic polymers, here chosen to be cellulose derivatives, we focus on thermodynamically stable homogeneous solutions. Thus the first step was to investigate the macroscopic behaviour of different mixed solutions. Figure 4 shows the phase maps for the $\mathrm{P}^{+} / \mathrm{dsDNA}$ and $\mathrm{HMP}^{+} / \mathrm{dsDNA}$ systems. The one-phase samples are homogeneous, some are bluish, others transparent, and often highly viscous gels. Most of the two-phase samples display two well-separated phases. Phase separation occurs readily for lower cationic polyelectrolyte contents. For the lowest concentrations of oppositely charged polymers (dsDNA and cationic polyelectrolyte), no macroscopic phase separation is detected. The most notable feature in these phase maps is the asymmetry with respect to charge stoichiometry (dashed line). One-phase regions occur when cationic polyelectrolyte is in excess, i.e., when there are more positive charges than negative ones. When a large amount of dsDNA is present in the system more cationic polyelectrolyte is required to reach the one-phase region. For the polyelectrolytes alone no phase separation was detected even up to high concentrations. Also we note that there are marked differences between the two-phase maps. We can observe that the two-phase and the bluish regions are much more extended in the $\mathrm{P}^{+} / \mathrm{dsDNA}$ system. The limit of the two-phase region for the $\mathrm{HMP}^{+} / \mathrm{dsDNA}$ system coincides closely with the neutralisation line.

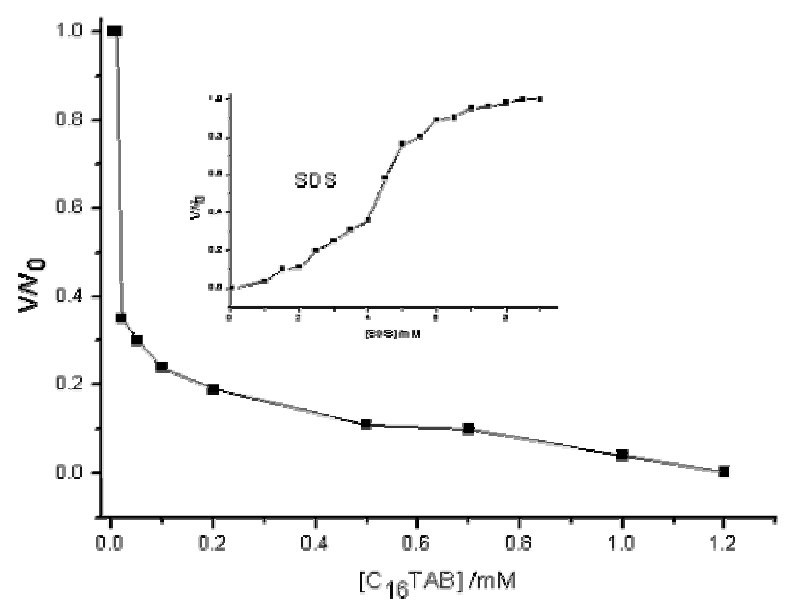

Figure 3. Swelling isotherm for chemical DNA gels (1\% cross-linker) immersed in solutions of the cationic surfactant $\mathrm{C}_{16} \mathrm{TAB}$ and thereafter in solutions of the anionic surfactant SDS.

The ssDNA systems show qualitatively the same behaviour. However, the interaction of $\mathrm{P}^{+}$ with ssDNA leads to a more extended two-phase region as compared to the $\mathrm{P}^{+} / \mathrm{dsDNA}$ system. 
The linear charge density of dsDNA ( $\sim 5.9$ negative charges $/ \mathrm{nm})$ is considerably higher than for ssDNA $(\sim 2.95$ negative charges/nm) and from a simple electrostatic mechanism dsDNA should interact more strongly with oppositely charged polyelectrolytes. However, the amphiphilic character is clearly very different for the two DNA states. In dsDNA, the hydrophobic groups are largely hidden while they are exposed to the solution for ssDNA. The expectation is that, in the latter case, hydrophobic interactions would be much more significant.

While further studies are required to understand phase separation and physical gel stability, the asymmetry deserves further comments. We thus see that only very minor additions of cationic polymers to DNA solutions lead to phase separation, while solutions of the cationic polymers are not phase separated on DNA addition until we are close to the charge neutralisation condition. One analogy that can be found to this behaviour is when cationic surfactant is added to DNA solutions; here phase separation occurs at very low surfactant concentrations, very far from charge stoichiometry.

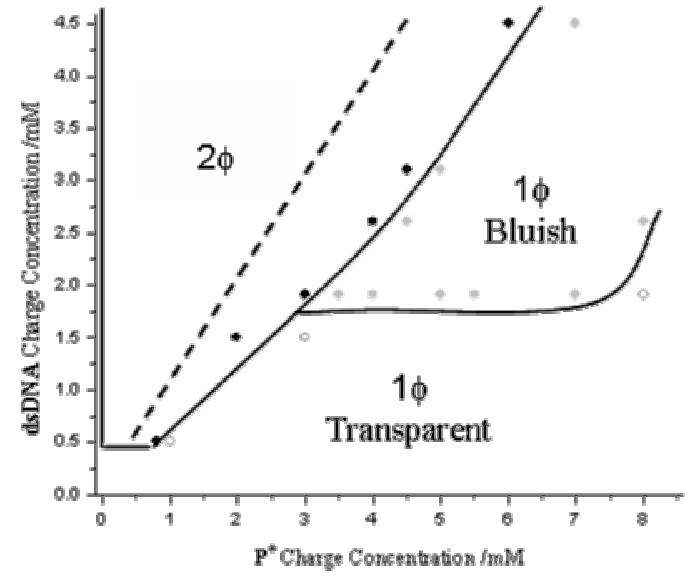

(a)

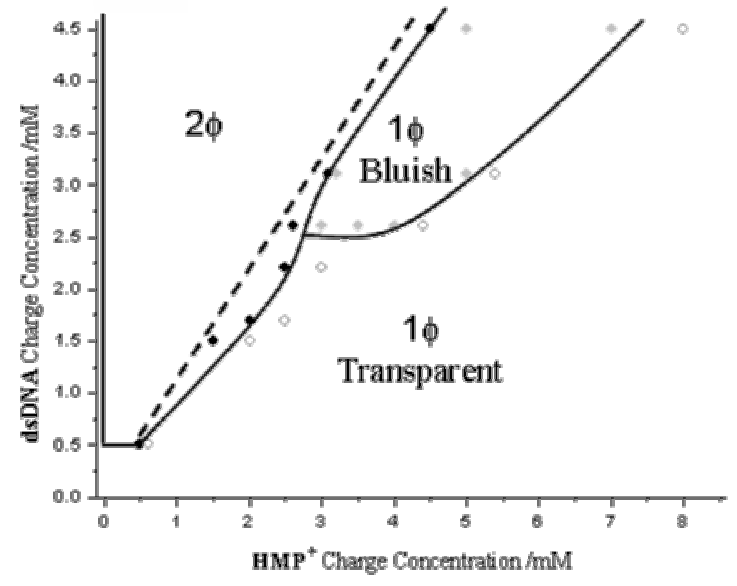

(b)

Figure 4. $\mathrm{P}^{+} / \mathrm{dsDNA}$ (a) and $\mathrm{HMP}^{+} / \mathrm{dsDNA}$ (b) phase maps. $2 \phi$ and $1 \phi$ denote the two-phase and the one-phase regions, respectively. dsDNA and cationic polyelectrolyte are presented in terms of charge concentration and the dashed line respresents the neutralisation line.

\section{Physical DNA gels-Rheology}

When the negatively charged dsDNA or ssDNA is added to a positively charged polyelectrolyte $\left(\mathrm{P}^{+}\right.$or $\left.\mathrm{HMP}^{+}\right)$solution, a very marked rise in viscosity is observed. The association between the two oppositely charged polyelecrolytes leads to an important enhancement in active links which confers a large elasticity to the system. These increases can be attributed to the electrostatic interactions in the $\mathrm{P}^{+} / \mathrm{dsDNA}$ system. The $\mathrm{HMP}^{+} / \mathrm{dsDNA}$ system it may not be the only interaction but also the hydrophobic tails may play a role.

In Figure 5 is presented the variation of the storage modulus, $\mathrm{G}^{\prime}$, as a function of the charge ratio, for different $\mathrm{P}^{+}$and dsDNA concentrations. As can be seen, over a wide range of 
compositions, the storage modulus has high values; thus we have an elastic behaviour. We also note the quite pronounced maximum at a certain composition with the maximum occurring in all cases at a charge ratio of $\sim 3.5$. It is striking that the location of the maximum does not change significantly with the total polymer concentration. At the maximum there are ten times more molecules of $\mathrm{P}^{+}$than dsDNA (one dsDNA molecule, $\sim 240 \mathrm{~nm}$ in length has 5.9 charges $/ \mathrm{nm}$ while a $\mathrm{P}^{+}$molecule has a mean contour length between charges of $2 \mathrm{~nm}$; the charge ratio $\mathrm{P}^{+} / \mathrm{dsDNA}$ is 0.35 ).

While a mechanism for this behaviour can only be established after further studies (including variations in molecular weight and investigations of aggregate structure), we can note that while the electrostatic interaction has a maximum at charge stochiometry, rheology can show a non-monotonic behaviour. Thus adding an oppositely charged polymer to a polyelectrolyte solution generally leads to an increased viscosity and storage modulus due to polymer-polymer association. This association is strengthened on approach of charge stoichiometry. However, approach to charge neutrality also leads to contraction of aggregates formed. At some point, the aggregates formed are unable to span the volume in a threedimensional network and then a decreased viscosity is expected. For a too strong association, a macroscopic phase separation occurs without extended networks and storage modulus vanishes. We note the clear correlation between the rheological behaviour and the phase maps and also the correlation between the eleastic behaviour and the bluish appearence of the samples, indicating large aggregates. It is clear that the gelation and the phase behaviour have common causes. It is significant that in comparative investigations of the storage and loss moduli as a function of frequency we have found that also the relaxation time has a maximum but not under the same conditions. This demonstrates that the stronger interaction (the long-lived network) does not coincide with the maximum of the number of cross-links. When the $\mathrm{P}^{+}$concentration is kept constant and the dsDNA content is varied, it can be inferred that the relaxation time, $\tau^{*}$, is higher for higher dsDNA concentrations.

For the $\mathrm{HMP}^{+} /$dsDNA system, $\mathrm{G}^{\prime}$ throughout increases with the $\mathrm{HMP}^{+}$concentration as well as with the dsDNA content. The increase with dsDNA is more pronounced. The association between the two oppositely charged polyelectrolytes leads to a great enhancement in active links which confers a large elasticity to the system. The association is much strengthened because of the combination of electrostatic and hydrophobic attraction. $\tau^{*}$ is higher for lower charge ratios. If a certain number of negative charges are present and positive charges are added to the system, the strongest interaction will be in or nearby the neutralisation situation. 


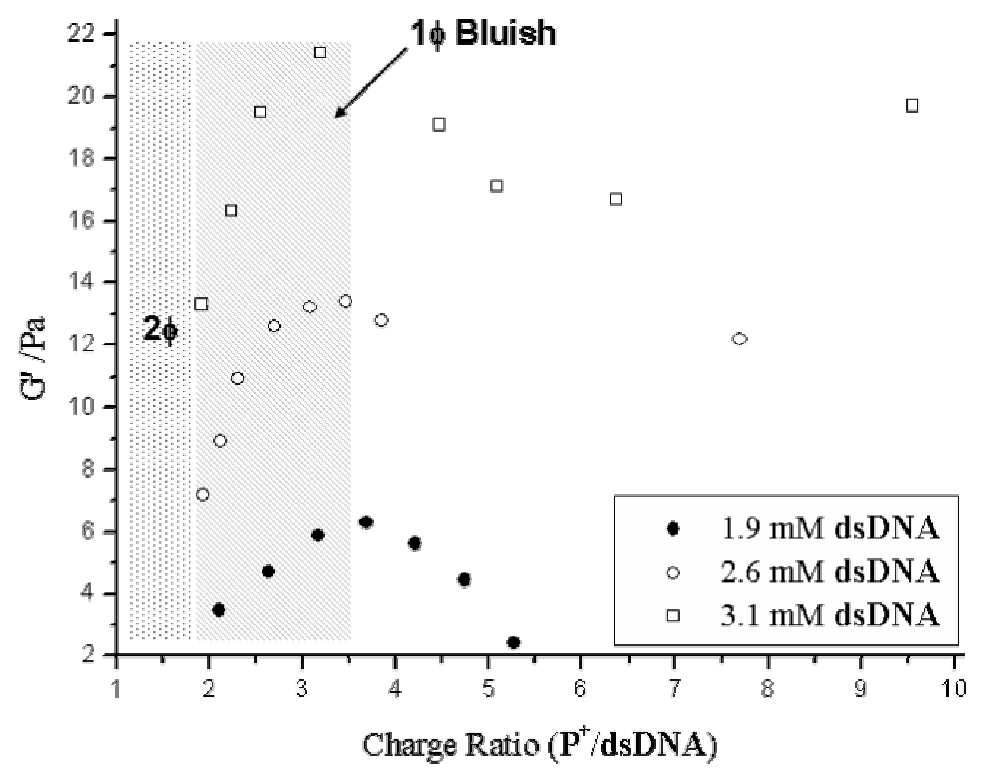

Figure 5. $\mathrm{G}^{\prime}$ as a function of charge ratio between $\mathrm{P}^{+}$and DNA. The viscosity follows the same tendency as $\mathrm{G}^{\prime}$. The behaviour of these rheological parameters with dsDNA concentration can also be seen. The oscillatory-shear measurements were obtained in a frequency range between $0.009 \mathrm{~Hz}$ and $90 \mathrm{~Hz}$.

In Figure 6, presenting a comparison between the ss and ds DNA systems, the general picture that emerges is that, for the same $\mathrm{P}^{+}$concentration, $\mathrm{G}^{\prime}$, complex viscosity $\eta^{*}$ and $\tau^{*}$ are higher for the $\mathrm{P}^{+} /$dsDNA system than for the $\mathrm{P}^{+} /$ssDNA system. With ssDNA, the system contains one cationic polyion and one anionic polyion which contains a hydrophobic core exposed to the solvent. Negative charges are the same in number but, compared to dsDNA, the charge density is reduced to half. As a consequence, $\mathrm{P}^{+}$and ssDNA establish weaker interactions.

For the $\mathrm{HMP}^{+} / \mathrm{ssDNA}$ system, $\mathrm{G}^{\prime}$ increases with both $\mathrm{HMP}^{+}$and ssDNA charge concentrations. An increase of the ssDNA concentration leads to a more pronounced raise of $\mathrm{G}^{\prime}$, i.e., the number of interactions (active links) increases strongly when more ssDNA molecules are put in the system. The systems with ssDNA, are composed of two amphiphilic polyelectrolytes and the interactions are electrostatic as well as hydrophobic. No conclusive differences are, however, obtained from the comparison between $\mathrm{G}^{\prime}$ and viscosity of the $\mathrm{HMP}^{+} / \mathrm{dsDNA}$ and $\mathrm{HMP}^{+} /$ssDNA systems. 


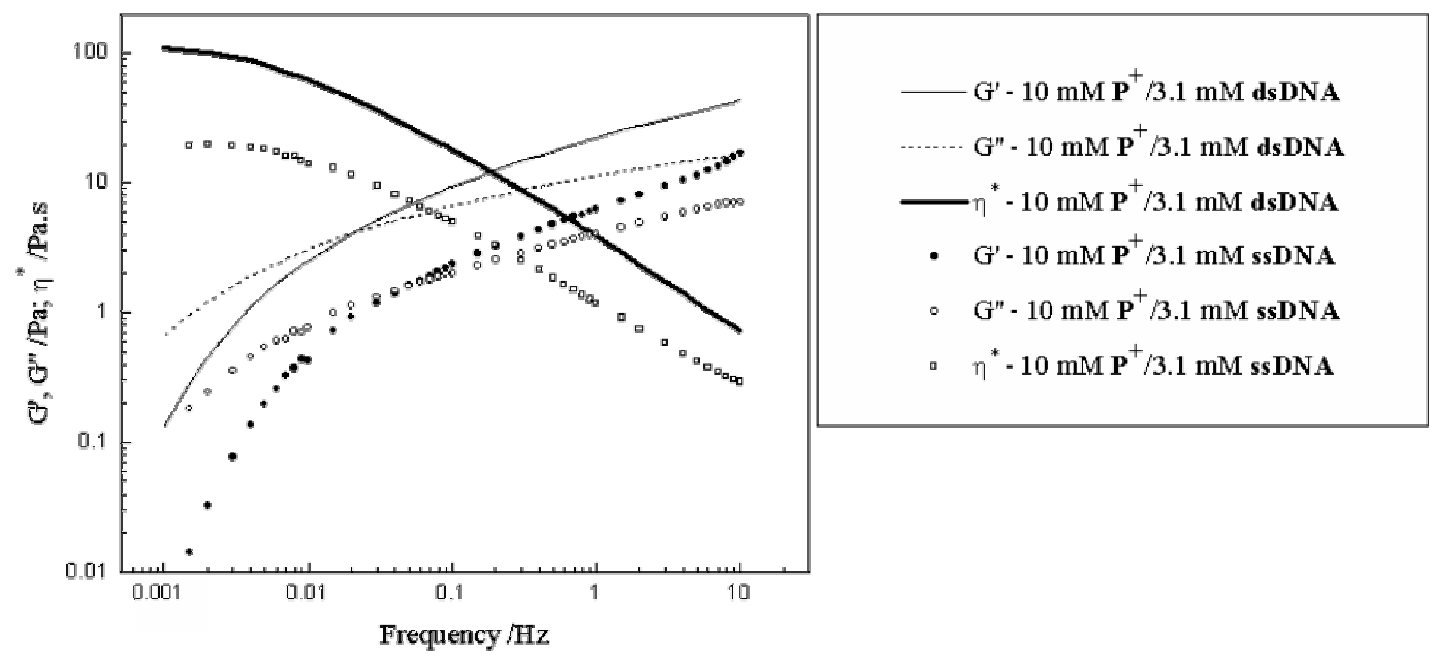

Figure 6. $\mathrm{G}^{\prime}, \mathrm{G}^{\prime \prime}$ and $\eta^{*}$, comparing the $\mathrm{P}^{+} / \mathrm{dsDNA}$ and $\mathrm{P}^{+} / \mathrm{ssDNA}$ systems. Rheological properties are presented as a function of frequency.

The $\tau^{*}$ is lower for the $\mathrm{HMP}^{+} / \mathrm{ssDNA}$ systems. However, for higher $\mathrm{HMP}^{+}$concentrations $\tau^{*}$ starts to present less dissimilar values. Possibly, the presence of electrostatic and hydrophobic interactions and their competition can lead to a more short-lived structure of $\mathrm{HMP}^{+} / \mathrm{ssDNA}^{2}$ systems. However, the trend of $\tau^{*}$ is the same for both $\mathrm{HMP}^{+} / \mathrm{ssDNA}$ and $\mathrm{HMP}^{+} / \mathrm{dsDNA}$ systems, i.e, it is higher when the two-phase region approaches.

\section{Conclusions}

The chemical DNA gels, obtained by covalent cross-linking of DNA chains, give an expected swelling/deswelling behaviour on addition of cosolutes and can establish themselves as an additonal possibility for monitoring DNA-cosolute interactions. The physical gels, obtained by cross-linking DNA chains by cationic polymers, show some novel features with respect to both stability and rheology. The intriguing asymmetries with respect to charge neutralisation will deserve further attention.

\section{Acknowledgements}

We acknowledged Bruno Medronho for all valuable discussions. The present work was supported by the Swedish Science Research Council (VR) and Fundação para a Ciência e a Tecnologia (FCT) (POCTI/QUI/45344/2002 and SFRH/BD/16736/2004). 


\section{References}

1. Carpenter, D. K. In Encyclopedia of Polymer Science and Technology; John Wiley \& Sons: New York, 1966; Vol.4, pp 16-73.

2. Kokufuta, E.; Suzuki, H.; Yoshida, R.; Yamada, K.; Hirata, M.; Kaneko, F. Langmuir 1998, $14,788$.

3. Lee, W.; Hsu, C. J. App. Polym. Sci. 1999, 74, 3242.

4. Eichenbaum, G. M.; Kiser, P. F.; Shah, D.; Meuer, W. P.; Needham, D.; Simon, S. A. Macromolecules 2000, 33, 4087.

5. Annaka, M.; Ogata, Y.; Nakahira, T. J. Phys. Chem. B 2000, 104, 6755.

6. Zhang, J.; Peppas, N. A. Macromolecules 2000, 33, 102.

7. Zhao, B.; Moore, J. S. Langmuir 2001, 17, 4758.

8. Sjöström, J.; Piculell, L. Langmuir 2001, 3836.

9. Sayil, C.; Okay, O. J. App. Polym. Sci. 2002, 83, 1228.

10. Kiser, P. F.; Wilson, G.; Needham, D. Nature 1998, 394, 459.

11. Akala, E. O.; Kopeckova, P.; Kopecek, J. Biomaterial. 1998, 19, 1037.

12. Torres-Lugo, M.; Peppas, N. A. Macromolecules 1999, 32, 6646.

13. Tanaka, T. "Hydrogels" In Encyclopedia of Polymer Science and Technology, Mark, H. F.: Kroschwitz, J. I., Eds, New York: Wiley, 1985; pp 514-531.

14. Horkay, F.; Tasaki, I.; Basser, P. J. Biomacrom. 2000, 1, 84.

15. Yodhida, R.; Uchida, K.; Kaneko, Y.; Sakai, K.; Kikuchi, A.; Sakurai, Y.; Okano, T. Nature 1995, 374, 240.

16. Gotoh, T.; Nakatani, Y.; Sakohara, S. J. J. Appl. Polym. Sci. 1998, 69, 895.

17. Lee, K. K.; Cussler, E. L.; Marchetti, M. Chem. Eng. Sci. 1990, 45, 766.

18. Ishihhara, K.; Muramoto, N.; Shinohara, I. J. Appl. Polym. Sci. 1984, 29, 211.

19. Irie, M. Adv. Polym. Sci. 1990, 94, 28.

20. Gehrke, S. H; Lee, P. I. "Hydrogels for drug delivery systems" In Specialized Drug Delivery Systems, Tyle P, Ed.; New York: Marcel Dekker, 1990; pp 333-392.

21. Eichenbaum, G. M.; Kiser, P. F.; Dobrynin, A. V.; Simon, S. A.; Needham, D. Macromolecules 1999, 32, 4867.

22. Kazanskii, K. S.; Dubrovskii, S. A. Adv. Polym. Sci. 1992, 97, 104.

23. Takezawa, T.; Mori, Y.; Yoshizato, K. Biotechnology 1990, 8, 854.

24. Liu, F.; Tao, G. L.; Zhuo, R. X. Polym. J. 1993, 25, 561.

25. Okano, T.; Kikuchi, A.; Sakurai, Y.; Takei, Y.; Ogata, N. J. Controlled Release 1995, 36, 125.

26. Mel'nikov, S. M., Mel'nikova, Y. S., Löfroth, J. J. Phys. Chem. B 1998, 102, 9367.

27. Mel'nikova, Y. S., Mel'nikov, S. M., Löfroth, J. Biophysical Chemistry 1999, 81, 125.

28. Köping-Höggård, M.; Mel'nikova, Y. S.; Vårum, K. M.; Lindman, B.; Artursson, P. J. Gene Med. 2003, 5, 130. 
29. Miguel, M. G., Pais, A. A. C. C., Dias, R. S., Leal, C., Rosa, M., Lindman, B. Colloids and Surfaces A: Physicochem. Eng. Aspects. 2003, 228, 43.

30. Dias, R. S., Pais, A. A. C. C., Miguel, M. G. J. Chem. Phys. 2003, 119, 8150.

31. Morris, E. R.; Ross-Murphy, S. B. In Techniques in Life Sciences, Vol. B 3, Elsevier/North Holland: Amsterdam, 1981.

32. Chen, Q.; Suki, B.; An, K. Summer Bioengineering Conference, Florida, 2003.

33. Costa, D.; Hansson, P.; Schneider, S.; Miguel, M.; Lindman, B. Biomacromol. In press.

34. Dias, R.; Lindman, B.; Miguel, M. J. Phys. Chem. B 2002, 106, 12608. 